\title{
Relationship Between Cycling Mechanics and Core Stability
}

\author{
John P. Abt, ${ }^{1}$ James M. Smoliga, ${ }^{1}$ Matthew J. Brick, ${ }^{2}$ John T. Jolly, ${ }^{3}$ Scott M. Lephart, ${ }^{1,4}$ \\ AND FREDDIE H. FU ${ }^{4}$
}

${ }^{1}$ Neuromuscular Research Laboratory, Department of Sports Medicine and Nutrition, University of Pittsburgh, Pittsburgh, Pennsylvania 15203; ${ }^{2}$ Millennium Institute of Sport and Health, Auckland, New Zealand; ${ }^{3}$ Center for Adaptive Neural Designs, The Biodesign Institute at Arizona State, Tempe, Arizona $85287{ }^{4}$ Department of Orthopedic Surgery, University of Pittsburgh, UPMC Center for Sports Medicine, Pittsburgh, Pennsylvania 15203.

\begin{abstract}
Abt, J.P., J.M. Smoliga, M.J. Brick, J.T. Jolly, S.M. Lephart, and F.H. Fu. Relationship between cycling mechanics and core stability. J. Strength Cond. Res. 21(4):1300-1304. 2007.-Core stability has received considerable attention with regards to functional training in sports. Core stability provides the foundation from which power is generated in cycling. No research has described the relationship between core stability and cycling mechanics of the lower extremity. The purpose of this study was to determine the relationship between cycling mechanics and core stability. Hip, knee, and ankle joint kinematic and pedal force data were collected on 15 competitive cyclists while cycling untethered on a high-speed treadmill. The exhaustive cycling protocol consisted of cycling at $25.8 \mathrm{~km} \cdot \mathrm{h}^{-1}$ while the grade was increased $1 \%$ every 3 minutes. A core fatigue workout was performed before the second treadmill test. Total frontal plane knee motion (test 1: $15.1 \pm 6.0^{\circ}$; test 2: 23.3 $\pm 12.5^{\circ}$ ), sagittal plane knee motion (test 1: $69.9 \pm 4.9^{\circ}$; test 2 : $79.3 \pm 10.1^{\circ}$ ), and sagittal plane ankle motion (test 1: $29.0 \pm$ $8.5^{\circ}$; test $2: 43.0 \pm 22.9^{\circ}$ ) increased after the core fatigue protocol. No significant differences were demonstrated for pedaling forces. Core fatigue resulted in altered cycling mechanics that might increase the risk of injury because the knee joint is potentially exposed to greater stress. Improved core stability and endurance could promote greater alignment of the lower extremity when riding for extended durations as the core is more resistant to fatigue.
\end{abstract}

KEY WoRDS. alignment, injury, overuse

\section{INTRODUCTION}

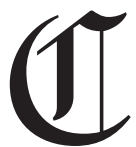

ore stability has received considerable attention with regards to functional training in sports. Anecdotally, core strengthening has been adopted by the athletic community and clinical professionals both for performance enhancement as well as injury prevention (17), with particular focus related to strengthening the abdominal, paraspinal, and gluteal muscles (11). The core not only provides stability to the spine while controlling movement at the torso, but also affords greater leverage for upper and lower extremity motion and force development (9).

Specific to cycling, the core provides the foundation from which force is generated (10). The core muscles maintain the neutral pelvic position on the bike when the anterior and posterior muscle components are equally balanced. On the basis of orientation and attachment of the psoas muscle on the lumbar spine, pelvic stabilization and resistance to fatigue are critical to maintain the natural curve of the spine as well as provide the leverage from which the psoas and gluteal muscles contract when a greater power output is required (8). Although the lack of core stability would appear to have the greatest influ- ence on the back, lower extremity alignment could also be affected as the foundation from which power is generated becomes compromised (2).

Appropriate bike fitting is critical to prevent injuries such that consistent lower extremity alignment is adopted throughout the pedal stroke (1). Decreased core strength however, could artificially induce malalignment of the lower extremity in an effort to maintain a given power output. Furthermore, the combined effect of lower extremity malalignment, excessive cadence, and increased riding volume or intensity could increase the risk of injury (1). The typical cyclist, with an average cadence of 90 revolutions per minute, will pedal between 16,000 and 21,000 revolutions during a typical 3-4-hour ride and upwards of 33,000 for a 6 -hour ride. The repetitive motion of cycling and the fixed position of the pelvis and feet require efficient movement patterns to avoid excessive stresses being applied to the musculoskeletal structures of the lower extremity (6).

Although the relationship between core stability and back injury in cyclists has been studied (3), no research that we are aware of has examined the role of core stability in maintaining lower extremity alignment during cycling. The core is often described according to the perspective of the investigator (17). For the purpose of this study, the core will refer to the main anterior and posterior lumbo-pevlic stabilizing muscles. The purpose of this study was to determine the relationship between cycling mechanics and core stability. It was hypothesized that diminishing core stability would result in altered cycling mechanics and pedal force application.

\section{Methods}

\section{Experimental Approach to the Problem}

A within-subject, repeated measures design was used to determine changes in lower extremity joint kinematics and pedaling forces as a result of compromised core stability. Subjects reported for 3 sessions (1 training, 2 testing) throughout the study. Sessions were separated by a minimum of 1 week to ensure full recovery and prevent potential confounding results on subsequent tests. An initial training session was provided to introduce subjects to testing methods. Subjects performed an incremental ramp cycling protocol during test 1 . Test 2 required the subjects to complete a precore fatigue isokinetic test, core fatigue workout, postcore fatigue isokinetic test, and a repeat of the incremental ramp protocol performed during test 1 . 


\section{Subjects}

Fifteen competitive cyclists (age: $34.5 \pm 9.8$ years; height: $1.77 \pm 0.11 \mathrm{~m}$; mass: $76.3 \pm 11.1 \mathrm{~kg}$ ) participated in this study. Subjects were members of local road cycling teams with a road race classification of category $2-4$ on the basis of racing experience and accumulated finishing place points by the U.S. Cycling Federation. Subjects provided written informed consent before participation in accordance with the university Institutional Review Board. Subjects who reported a history of musculoskeletal injury within the previous 3 months or participated in a core strengthening program ( 2 or more times per week for 6 weeks before study enrollment) on a regular basis were excluded.

\section{Procedures}

Training Session. Subjects were provided a separate training session to become familiar with the testing procedures and, specifically, riding on the treadmill (Woodway ELG; Woodway USA, Waukesha, WI). Proper gear ratio was determined during the training session to ensure subjects were able to maintain the desired pace with a cadence of 90-95 revolutions per minute. This would be the gear ratio that subjects would ride during the testing sessions.

Incremental Ramp Protocol-Test 1 and Test 2. One week after the training session, subjects reported for test 1. Linear and circumferential anthropometric measurements of the dominant lower extremity were recorded for each subject. Spherical reflective markers (diameter $0.025 \mathrm{~m}$ ) were placed at designated anatomical landmarks previously described by Vaughan et al. (16). Two additional markers were positioned at the most lateral aspect of the pedal in line with the pedal spindle and approximately $4.5 \mathrm{~cm}$ inferior to the pedal spindle marker. Raw coordinate data were collected with the Peak Motus 3D Motion Analysis System (software version 7.1; ViconPeak Inc., Centennial, CO) interfaced with 8 highspeed $(120-\mathrm{Hz})$ optical cameras (Pulnix Industrial Product Division, Sunnyvale, CA). Dependent kinematic variables included total frontal and sagittal plane motion of the hip and knee and total sagittal plane motion of the ankle. Intraclass correlation coefficients (ICCs) and standard error of measurement (SEM) were previously calculated within our laboratory for test-retest reliability of all kinematic measurements (ICC 0.843-0.957; SEM $\left.0.97-1.89^{\circ}\right)$.

Raw force data $(1,200 \mathrm{~Hz})$ were collected with the use of custom-designed pedals constructed from silicone strain gauge force transducers (ATI Industrial Automation, Apex, NC) and Shimano Pedaling Dynamics (SPD) pedals (Shimano, Osaka, Japan). A local coordinate system was created on the bicycle, with the origin positioned on the seat tube, $5 \mathrm{~cm}$ inferior to the seat tube-top tube junction. Two additional markers were positioned on the down tube at the same vertical height as the origin marker and the center of the head tube. The local $y$-axis was calculated as the vector between the seat tube and down tube markers, whereas the $x$-axis was formed by the vector between the seat tube and head tube markers. The cross product of the $x$ - and $y$-axes was used to calculate the $z$-axis. Dependent variables included power phase effective force, recovery phase effective force, total gross work, total net work, positive work, and negative work. Power phase was defined as the time period that corresponded to $0-180^{\circ}$ of the pedal stroke, and recovery phase was defined as the time period that corresponded to 180 $360^{\circ}$ of the pedal stroke. Coefficient of variation for pedal force data has been previously determined within our laboratory to be $18.3-24.4 \%$. Coefficient of variation for work data has been previously determined within our laboratory to be $20.7-23.2 \%$.

Subjects rode their own bikes and wore their own cycling shoes with SPD cleats (Shimano, SM-SH51) to ensure natural position within the SPD pedal (Shimano, PD-M520). Subjects were provided a 10-minute warm-up before data collection. The treadmill protocol for test 1 and test 2 consisted of riding untethered on a high-speed treadmill at $25.8 \mathrm{~km} \cdot \mathrm{h}^{-1}$. The treadmill elevation was increased in 1\% increments every 3 minutes until exhaustion. Subjects were required to maintain the same gear ratio, cadence, and hand position throughout the test while remaining seated. A total of 7 pedal cycles were collected during the final 30 seconds of each stage, with the middle 3 trials being used for data analysis.

Isokinetic Torso Rotation Test-Test 2. The Biodex System 3 Multi-Joint Testing and Rehabilitation System (Biodex Medical Inc., Shirley, NY) was used to validate core fatigue following the core fatigue workout by determining the changes in torque, work, and power after the exercise circuit. Subjects were seated in an upright position with their popliteal space approximately $6 \mathrm{~cm}$ from the edge of the seat of the chair. The torso rotation attachment was aligned with the long axis of the spine and lowered to contact the subject's chest approximately $4 \mathrm{~cm}$ below the level of the clavicles. Practice trials were provided to ensure patient understanding and familiarity. Subjects were instructed to perform maximal intensity, concentric isokinetic axial torso rotations at $120^{\circ} \cdot \mathrm{s}^{-1}$ for 3 minutes without pacing. Right and left rotational data were averaged for peak torque, total work, average power, maximum repetition total work, and average peak torque per repetition.

Core Fatigue Workout-Test 2. Subjects performed a 32 minute circuit of 7 exercises designed to target core stabilizer muscles in multiple planes of motion. Each subject completed 4 consecutive sets of the exercise circuit, performing each exercise for 40 seconds and resting for 20 seconds. The exercise circuit consisted of the following exercises: seated upper torso rotations with medicine ball, static prone torso extension with medicine ball, supine lower torso rotations with medicine ball, incline sit-ups with weighted plate, lateral side bend (performed bilaterally) with weighted plate, rotating lumbar extension with weighted plate, and standing torso rotations with weighted pulley resistance.

Immediately after the exercise circuit, subjects performed a second isokinetic torso rotation test to verify fatigue of the core musculature. On completion of the postexercise isokinetic test, subjects performed a second incremental cycling treadmill test as described for test 1 .

Data Reduction. Raw coordinate data were filtered with a fourth-order Butterworth filter with an optimal cutoff frequency (7). All of the kinematic calculations were performed in the Kincalc module of the Peak Motus software package and based on Vaughan et al. (16). Raw force data were filtered with a fourth-order Butterworth filter with an optimal cutoff frequency (7). The filtered coordinate and analog data were then exported to a custom-designed LabView (version 6; National Instruments, Austin, TX) program to calculate the joint kinematic and pedal force data of interest.

Initially, the pedal markers were converted to the lo- 
TABLE 1. Precore fatigue and postcore fatigue isokinetic torso rotation data.

\begin{tabular}{lcc}
\hline & Precore fatigue & Postcore fatigue \\
\hline Peak torque $(\mathrm{N} \cdot \mathrm{m})$ & $96.8 \pm 35.0$ & $67.4 \pm 24.9^{*}$ \\
Total work $(\mathrm{J})$ & $6,716.9 \pm 2,394.5$ & $4,276.6 \pm 1,517.0^{*}$ \\
Average power $(\mathrm{W})$ & $74.9 \pm 26.7$ & $46.6 \pm 16.4^{*}$ \\
Peak repetition total work $(\mathrm{J})$ & $125.9 \pm 50.6$ & $71.4 \pm 29.7^{*}$ \\
Average peak torque $(\mathrm{N} \cdot \mathrm{m})$ & $68.7 \pm 22.8$ & $48.1 \pm 17.0^{*}$ \\
\hline
\end{tabular}

* Significant prefatigue/postfatigue difference $(p<0.05)$.

cal coordinate system of the bike. The average position of the pedal spindle marker was used to calculate the center of rotation of the crank and the vector from the crank center of rotation to the pedal spindle representing crank length. The vector from the inferior pedal spindle marker to the center pedal spindle marker represented the orientation of the pedal with respect to the crank vector. The pedal forces were initially calculated in their own orientations and then transformed to coincide with the local coordinate system of the bike. Forces in the bike local coordinate system were then transformed to an instantaneous coordinate system coinciding with the circular motion of the bike crank. The tangential force component axis was aligned along the fore/aft direction of the pedal, the normal force component axis was aligned perpendicular to the pedal, and the mediolateral force component was calculated as the cross product of the tangential and normal force directions to coincide with the mediolateral axis of the bike local coordinate system. The effective force (work perpendicular to crank) and ineffective force (work parallel to the crank) components were calculated from the normal and tangential pedal components and as force vectors relative to crank angle (1).

Instantaneous angular work was calculated throughout each crank cycle as the product of the effective force and distance of rotation (arc length $=$ crank length $\times$ angular displacement). Total positive work and total negative work were initially calculated as the area under the instantaneous work and crank angle curve. Net work was calculated as the difference between positive and negative work. Gross work was calculated as the sum of the absolute values of positive work and negative work.

\section{Statistical Analyses}

SPSS 11.5 (SPSS, Inc., Chicago, IL) was used for all statistical procedures. Separate dependent $t$-tests were initially used to analyze differences for isokinetic torso rotation variables (peak torque, total work, average power, maximum repetition total work, and average peak torque per repetition) after the core fatigue workout. Separate dependent $t$-tests were used to examine kinematic (total frontal and sagittal plane motion of the hip and knee and total sagittal plane motion of the ankle) and force (power phase effective force, recovery phase effective force, total gross work, total net work, positive work, negative work) differences of the final stage attained between test 1 and test 2. Statistical significance was set a priori at $p \leq 0.05$ for all analyses.

\section{Results}

A significant decrease (30.0-43.3\%) in peak torque, total work, average power, maximal repetition total work, and average peak torque was demonstrated after the core fatigue workout, confirming the effect of the core fatigue workout to induce fatigue. Isokinetic torso rotational data are presented in Table 1 .
TABLE 2. Test 1-test 2 kinematic data.

\begin{tabular}{lll}
\hline & \multicolumn{1}{c}{ Test 1} & \multicolumn{1}{c}{ Test 2} \\
\hline Hip sagittal plane motion $\left(^{\circ}\right)$ & $47.1 \pm 7.1$ & $48.0 \pm 5.1$ \\
Hip frontal plane motion $\left(^{\circ}\right)$ & $17.7 \pm 15.7$ & $14.4 \pm 7.2$ \\
Knee sagittal plane motion $\left(^{\circ}\right)$ & $69.9 \pm 4.9$ & $79.3 \pm 10.1^{*}$ \\
Knee frontal plane motion $\left(^{\circ}\right)$ & $15.1 \pm 6.0$ & $23.3 \pm 12.5^{*}$ \\
Ankle sagittal plane motion $\left(^{\circ}\right)$ & $29.0 \pm 8.5$ & $43.0 \pm 22.9^{*}$ \\
\hline
\end{tabular}

* Significant test 1 -test 2 difference $(p<0.05)$.

TABLE 3. Test 1-test 2 pedal force and work data.

\begin{tabular}{lrr}
\hline & \multicolumn{1}{c}{ Test 1} & \multicolumn{1}{c}{ Test 2} \\
\hline Power effective force $(\mathrm{N})$ & $354.6 \pm 75.3$ & $355.8 \pm 63.3$ \\
Recovery effective force $(\mathrm{N})$ & $57.1 \pm 38.6$ & $60.5 \pm 23.7$ \\
Gross total work $(\mathrm{J})$ & $127.6 \pm 24.8$ & $126.8 \pm 20.7$ \\
Net total work $(\mathrm{J})$ & $100.5 \pm 22.2$ & $100.9 \pm 23.2$ \\
Positive work $(\mathrm{J})$ & $114.8 \pm 22.5$ & $114.7 \pm 21.0$ \\
Negative work $(\mathrm{J})$ & $12.8 \pm 7.5$ & $12.1 \pm 6.2$ \\
\hline
\end{tabular}

Because of variability in completion of the incremental ramp protocol between subjects, kinematic and force data are reported at the final stage as subjects self-terminated the test because of exhaustion. Termination of test 1 and test 2 were completed within the same stage within subjects $(5 \pm 1 \%)$. Total frontal plane knee motion and total sagittal plane knee and ankle motion increased (13.4$54.3 \%$ ) after the core fatigue protocol, indicating greater extraneous motion throughout the pedal stroke. Kinematic data are presented in Table 2 .

No significant differences were demonstrated for any pedal force or work data. Pedal force and work data are presented in Table 3.

\section{Discussion}

The purpose of this study was to determine the relationship between cycling mechanics and core stability. The results of this study only partially supported our hypotheses. Specifically, several of the kinematic variables were altered after the core fatigue workout, whereas the pedal force and work variables remained unchanged. Collectively, these results would suggest compensatory kinematic adaptations to maintain a given power output.

Knee pain remains one of the most commonly diagnosed pathologies in cyclists, with factors such as bicycle fit, improper training, and anatomical abnormalities identified as contributors to injury (6). Considering the fixed pelvis and feet positions, the knee acts as the fulcrum of the thigh and shank, at which point excessive stresses are likely to be absorbed. Cycling mechanics typically involve a pistonlike, symmetrical motion of the legs for power generation and smooth rolling transition between the contact points of the patellofemoral joint $(4,5)$. Disrupted tracking of the patella could result in wearing on the posterior surface of the patella because of excessive 
frontal plane motion of the knee (5). As identified in this study, disruption of core stability resulted in greater total frontal plane knee motion and altered the cyclical, aerodynamic position of knees near the top tube with a greater valgus positioning toward the top tube. The subjects also displayed a combination of greater total sagittal plane knee motion and total sagittal plane ankle motion. The adopted sagittal plane knee motion pattern could have been a compensatory adaptation as a result of ankling to increase the leverage of the foot against the pedal. The lack of core stability might amplify the influence of the other factors (strength imbalances, flexibility deficits, heavy gear selection, large accumulation of miles) that are known to contribute to knee pathology (4), particularly as cyclists continue to ride for durations of several hours with altered mechanics of the lower extremity.

The application of pedaling forces has been previously studied in normal $(12,13)$ and prolonged cycling conditions (14). The typical pedaling stroke is separated into the effective (force application perpendicular to crank arm) and ineffective (force application parallel with crank arm) components (1). By partitioning the application of pedal forces into the respective components, inefficient force patterns can be identified, particularly as the feet transition through the top and bottom of the pedal stroke (1). Inefficient force patterns include persistent inferior vertical force at the bottom of the pedal stroke and insufficient superior forces during the recovery phase as the top of the pedal stroke approaches.

The role of core stability and subsequent changes in pedaling forces has received limited attention. Sanderson and Black (14) demonstrated an improvement in pedaling effectiveness during the power phase coupled with a reduction in pedaling effectiveness during the recovery phase as a result of prolonged cycling. The authors contended that the increased power phase effective force was a compensatory mechanism to offset the diminished effective force during the recovery phase (14). The lack of changes in pedaling forces, specifically during the recovery phase, was somewhat surprising considering our hypothesis that disrupting core stability would result in a reduction in pedaling effectiveness during the recovery phase. The lack of differences between test 1 and test 2 for positive work, negative work, net work, and gross work was also not expected. On the basis of our hypotheses, improved pedaling efficiency would have been demonstrated with decreased effective force during the recovery phase or a reduction in negative work. However, considering the response to the pedaling forces after the core fatigue workout, the work data followed a similar pattern and was consistent between the 2 treadmill tests. The fixed speed of the treadmill might have forced the subjects to maintain a similar pedaling efficiency (effective forces, work) between tests in this study in an effort to continue riding at the dictated pace, despite diminished core stability. Combined with the altered knee and ankle kinematic data, it is likely that core fatigue would have resulted in greater power phase effective force and less recovery phase effective force had a manual treadmill or roller system been used to better replicate the environment encountered during daily outside riding because the pace or resistance could be changed freely by the subject. The work of Sanderson and Black (14) further supports the notion that the treadmill speed might have influenced the test results because no changes were demonstrated for either the power phase or recovery phase effective forces despite the kinematic changes. Similarly, Sarre et al. (15) demonstrated greater positive and negative work during pedaling when the cadence increased.

Several limitations have been recognized by the authors. The fixed speed and gear ratio within and between tests might have negated the likelihood of finding significance with the pedaling force data. The inability to shift to an easier gear or slow down could have forced the subjects to pedal in a manner that resulted in the same performance, despite the altered mechanics of the lower extremity. Combined with the fixed treadmill speed, it is understandable that no changes were present for the pedal force data because similar pedaling efficiency was required to maintain the same position on the treadmill. The lack of changes in pedal force data further supports the importance of the core in lower extremity cycling mechanics in that the altered kinematic patterns were likely compensatory adaptations to maintain similar force patterns. However, the changes in biomechanical variables indicated that the use of untethered treadmill cycling forced the cyclists to adopt similar stabilizing mechanisms as experienced with outdoor, natural cycling.

The core fatigue protocol used in this investigation resulted in a significant reduction in core strength, endurance, and lower extremity alignment as verified by the isokinetic tests and the cycling treadmill tests. Furthermore, the cycling treadmill test of test 2 was started within 5 minutes of completing the isokinetic test to prevent recovery from the induced core fatigue. Although the targeted and acute core fatigue induced during the current study did not evolve in the progressive, low-intensity manner that would occur during natural cycling, the lack of core stabilization as a result of fatigue or weakness might produce a similar outcome regardless of mechanism.

The results of this study suggest that core stability contributes to lower extremity cycling mechanics. Improvements in core strength could promote greater torso stability within the saddle and maintenance of lower extremity alignment to apply greater force transmission to the pedals. Future research should examine the role of core fatigue on muscle activity of the specific core muscles, joint mechanics, and pedaling forces during unrestricted, self-pacing cycling that better simulates outdoor riding. Finally, future research should be performed to develop cycling-specific training programs to improve core strength and to determine whether such training programs reduce fatigue-related changes in cycling mechanics. Collectively, these data could be used to determine how core training most effectively influences performance and reduces the risk of injury in cyclists.

\section{Practical Applications}

The introduction of a core fatigue workout altered the mechanics of the lower extremity during cycling while pedal force application remained unchanged. Prolonged cycling with altered lower extremity mechanics as a result of a fatigued core might increase the risk of overuse injury from malalignment. Cyclists should integrate a yearround core conditioning program into current training to promote lower extremity alignment while cycling. Although cycling is primarily a sagittal plane activity, a core conditioning program should incorporate both sagittal and frontal plane exercises. Strengthening the core musculature could enhance the stability of the foundational leverage from which the cyclist generates power, and increasing the endurance of the core muscles could promote core stability maintenance. 


\section{REFERENCES}

1. Burke, E.R. High-Tech Cycling. Champaign, IL: Human Kinetics, 1996.

2. BuRKe, E.R. Serious Cycling. Champaign, IL: Human Kinetics, 2002.

3. Burnett, A.F., M.W. Cornelius, W. Dankaerts, and B.P. O'Sullivan. Spinal kinematics and trunk muscle activity in cyclists: A comparison between healthy controls and non-specific chronic low back pain subjects-A pilot investigation. Manual Ther. 9:211-219. 2004.

4. Faria, E.W., D.L. Parker, AND I.E. Faria. The science of cycling: Physiology and training-Part 1. Sports Med. 35:285-312. 2005.

5. FARIA, I.E. Energy expenditure, aerodynamics and medical problems in cycling. An update. Sports Med. 14:43-63. 1992.

6. Holmes, J.C., A.L. Pruitt, AND N.J. Whalen. Lower extremity overuse in bicycling. Clin. Sports Med. 13:187-205. 1994.

7. JACKSON, K.M. Fitting of mathematical functions to biomechanical data. IEEE Trans. Biomed. Eng. 26:122-124. 1979.

8. Juker, D., S. MCgill, P. Kropf, ANd T. Steffen. Quantitative intramuscular myoelectric activity of lumbar portions of psoas and the abdominal wall during a wide variety of tasks. Med. Sci. Sports Exerc. 30: 301-310. 1998.

9. Kibler, W.B., J. Press, ANd A. Sciascia. The role of core stability in athletic function. Sports Med. 36:189-198. 2006.

10. Mellion, M.B. Neck and back pain in bicycling. Clin. Sports Med. 13: 137-164. 1994.
11. Nadler, S.F., G.A. Malanga, L.A. Bartoli, J.H. Feinberg, M. PrybiCIEN, AND M. DEPRINCE. Hip muscle imbalance and low back pain in athletes: Influence of core strengthening. Med. Sci. Sports Exerc. 34:916. 2002.

12. Patterson, R.P., AND M.I. Moreno. Bicycle pedaling forces as a function of pedaling rate and power output. Med. Sci. Sports Exerc. 22:512-516. 1990.

13. RedField, R., AND M.L. Hull. On the relation between joint moments and pedaling rates at constant power in bicycling. J. Biomech. 19:317329. 1986.

14. SANDERSON, D.J., AND A. Black. The effect of prolonged cycling on pedal forces. J. Sports Sci. 21:191-199. 2003.

15. Sarre, G., R. Lepers, and J. Van Hoecke. Stability of pedaling mechanics during a prolonged cycling exercise performed at different cadences. J. Sports Sci. 23:693-701. 2005.

16. Vaughan, C.L., B.L. Davis, AND J.C. O'Connor. Dynamics of Human Gait. Western Cape, South Africa: Kiboho Publishers, 1991.

17. Wilson, J.D., C.P. Dougherty, M.L. Ireland, and I. McClay Davis. Core stability and its relationship to lower extremity function and injury. J. Am. Acad. Orthop. Surg. 13:316-325. 2005.

Address correspondence to John P. Abt, jpast16@pitt. edu. 